\title{
Reintervenção endodôntica em dente com canal em forma de C: Relato de caso
}

\section{Endodontic reintervention in tooth with c-shaped canal: Case report}

Fabio Luiz Soares ${ }^{1}$, Sammila Oliveira Faria ${ }^{2}$, Rogenilson Taylor Pereira Moura ${ }^{3}$

\section{RESUMO}

Introdução: Os canais em forma de C apresentam-se como variações anatômicas encontradas principalmente em segundos molares inferiores caracterizadas pela presença de um canal em forma de fita. Devido a sua complexidade e imprevisibilidade anatômica, a intervenção ou reintervenção endodôntica exige do profissional o planejamento e condução cuidadosa do tratamento. O objetivo deste estudo é relatar um caso clínico envolvendo a reintervenção endodôntica em dente com canal em forma de C. Relato de Caso: Paciente foi encaminhado ao consultório odontológico para a realização de reintervenção endodôntica do dente 37 . O exame radiográfico mostrou a presença de tratamento endodôntico parcial e rarefação periodontal estendendo da margem óssea distal até o periápice. Foi realizado o preparo químico mecânico e utilizado medicação intracanal à base de hidróxido de cálcio. Após 30 dias foi efetuado a obturação do sistema de canais radiculares. Proservações foram realizadas após 45 dias, 75 dias, 120 dias e 17 meses. Os exames radiográficos evidenciaram a redução da rarefação periapical com total formação óssea após 17 meses. Conclusão: Após 17 meses de proservação foi possível observar o reparo ósseo associado a ausência de sinais e sintomas.

Palavras-chave: Reintervenção Endodôntica. Retratamento endodôntico. Canal em forma de C

\section{ABSTRACT}

Introduction: C-shaped canals appear as anatomical variations found mainly in lower second molars characterized by the presence of a ribbon-shaped channel. Due to its complexity and anatomical unpredictability, endodontic intervention or reintervention requires the professional to plan and conduct the treatment carefully. The aim of this study is to report a clinical case involving endodontic reintervention in a tooth whith Cshaped canal. Case Report: The patient was referred to the dental office for endodontic reintervention of tooth 37 . The radiographic examination showed the presence of partial endodontic treatment and periodontal rarefaction extending from the distal bone margin to the peripexix. Mechanical chemical preparation was carried out and intracanal medication based on calcium hydroxide was used. After 30 days, the root canal system was filled. Follow-up were performed after 45 days, 75 days, 120 days and 17 months. Radiographic exams showed a reduction in periapical rerefaction with total bone formation after 17 months. Conclusion: After 17 months of follow-up, it was possible to observe bone repair associated with the absence of signs and symptoms.

Keywords: Endodontic reintervention. Endodontic retreatment. C-shaped canal.

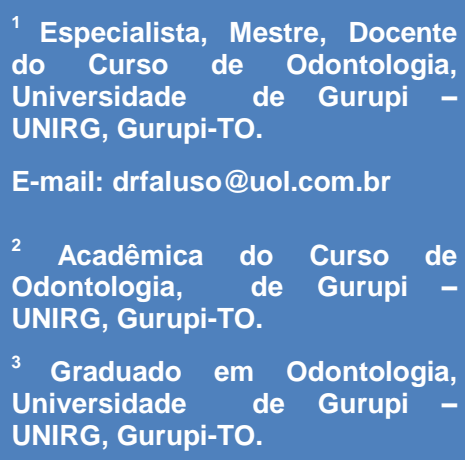

1 Especialista, Mestre, Docente do Curso de Odontologia, Universidade de Gurupi UNIRG, Gurupi-TO.

E-mail: drfaluso@uol.com.br 


\section{INTRODUÇAOO}

Canais em forma de C são todos aqueles que apresentam esboço geral de um "C" e presente em uma raiz em forma de $\mathrm{C}$, ou seja, com um sulco longitudinal na superfície da raiz, independentemente se um canal ou orifício separado foi observado ${ }^{1}$. Foram descritos inicialmente em 1979 por Cooke e Cox ${ }^{2}$. De acordo com Al-Fouzan ${ }^{1}$ para os segundos molares inferiores serem qualificados como tendo um sistema de canal em forma de $\mathrm{C}$ tiveram que apresentar raízes fusionadas, um sulco longitudinal lingual ou vestibular da raiz e pelo menos uma seção transversal do canal pertencente a configuração C1, C2 ou C3, modificada de Melton et al. ${ }^{3}$.

Al-Fouzan ${ }^{1}$ classificou os canais em forma de $C$ de acordo com os seguintes critérios: Categoria I: canal contínuo em forma de $C$ que sai da câmara pulpar para o ápice. Categoria II: orifício em forma de 'ponto e vírgula' (;) no qual a dentina separava um canal principal em forma de $\mathrm{C}$ de um canal mesial distinto

Categoria III: subdivisão I, orifício em forma de $\mathrm{C}$, no terço coronal, dividido em dois ou mais canais separados que se uniram apicalmente; subdivisão II, orifício em forma de C no terço coronal que dividido em dois ou mais canais discretos e separados no terço médio ao ápice; e subdivisão III, orifício em forma de $\mathrm{C}$, dividido em dois ou mais canais discretos e separados no terço coronal ao ápice

Woelber et al. ${ }^{4}$ avaliando um grupo de 901 molares tratados encontraram 15 dentes com uma configuração de canal radicular em forma de $C(1,66 \%)$, considerando os 160 segundos molares mandibulares, 6,87\% apresentaram essa configuração. Em seu estudo Al-Fouzan ${ }^{1}$ observou uma taxa de $10,6 \%$ de canais em formas de $C$ em segundos molares inferiores. Em estudo de Haddad et al. ${ }^{5}$ 19,1\% dos segundos molares examinados apresentaram canais em forma de $\mathrm{C}$. Utilizando tomografia computadorizada Jin et al. ${ }^{6}$ observaram que 98 dentes de 220 segundos molares inferiores apresentavam canais em forma de C $(44,5 \%)$. Embora a prevalência de canais em forma de C é significativamente maior nos segundos molares inferiores, também são encontrados em pré-molares inferiores, mas em pequenas porcentagens ${ }^{7}$. Mashyakhy et al. ${ }^{7}$ observaram em seu estudo que 29 dos 367 segundos molares (7,9\%) exibiram essa morfologia em forma de $C$, enquanto em pré-molares a prevalência de canais em forma de $C$ foi de $1,5 \%$ e $0,8 \%$ no primeiro e no segundo pré-molares, respectivamente 
Cooke e Cox $^{2}$ relataram que radiografias pré-operatórias e anatomias das câmaras pulpares indicam anatomia normal dos canais em segundos molares inferiores. Entretanto em estudo de Haddad et al. ${ }^{5}$ quase todas as radiografias pré-operatórias mostraram características que formaram uma imagem típica que permitia previsão da existência dessa condição anatômica tais como fusão radicular ou proximidade, um grande canal distal, canal mesial estreito e imagem embotada de um terceiro canal intermediário. Estudo de Fan et al. ${ }^{8}$ com objetivo de investigar a relação entre as características radiográficas dos segundos molares inferiores em forma de $\mathrm{C}$ e as formas transversais do sistema de canais radiculares sugeriu que a presença e a anatomia interna do sistema de canais em forma de $\mathrm{C}$ nos segundos molares inferiores podem ser previstas de acordo com a aparência radiográfica.

Dentes com canais em forma de C que requerem tratamento endodôntico apresentam um desafio para o clínico. Não apenas os procedimentos normais para o preparo do canal as vezes são inadequados, mas a presença de uma alta incidência de anastomoses transversais, canais laterais e deltas apicais dificulta a limpeza e a obturação adequada do sistema de canais ${ }^{9}$.

Exames de reconstruções 3D após o procedimento de obturação do canal e a etapa final do retratamento mostraram resíduos de materiais obturadores em todos os dentes. Aparentemente, eles foram deixados para trás em áreas inacessíveis à instrumentação. Frequentemente os remanescentes de material obturador foram transportados para locais do canal radicular não preenchidos anteriormente, como reentrâncias e ramificações ${ }^{10}$. Há de se considerar que devido à complexidade $\mathrm{e}$ imprevisibilidade anatômica de dentes em forma de C o completo preparo químico cirúrgico e selamento tridimensional do sistema de canais radiculares torna-se um fator que dificulta consideravelmente o tratamento. Frente a isso, em casos de insucesso, a reintervenção, quando possível, é a primeira opção de ser realizada. Neste contexto, o objetivo deste estudo é relatar um caso clínico onde apresentamos a conduta realizada em reintervenção em dente com canal em forma de $C$ e sua devida proservação.

\section{RELATO DE CASO}

O presente artigo trata de relato de caso clínico, de forma descritiva, com abordagem observacional. 
Paciente leucoderma, gênero masculino, 34 anos de idade, foi encaminhado ao consultório odontológico para a realização de reintervenção endodôntica do dente 37. Após a anamnese, ao exame clínico, onde foi observado a presença de restauração metálica fundida no dente em questão. Realizados os exames de palpação e percussão não foi observada qualquer alteração. Ao exame periodontal foi detectada a presença de bolsa periodontal com profundidade de $5 \mathrm{~mm}$ na distal do dente 37 .

O exame radiográfico sugeriu a fusão radicular mostrando a presença de tratamento endodôntico parcial com ausência de canal tratado, bifurcação em terço médio de raiz no canal distal e rarefação periodontal estendendo da margem óssea distal até o periápice (Figura 1).

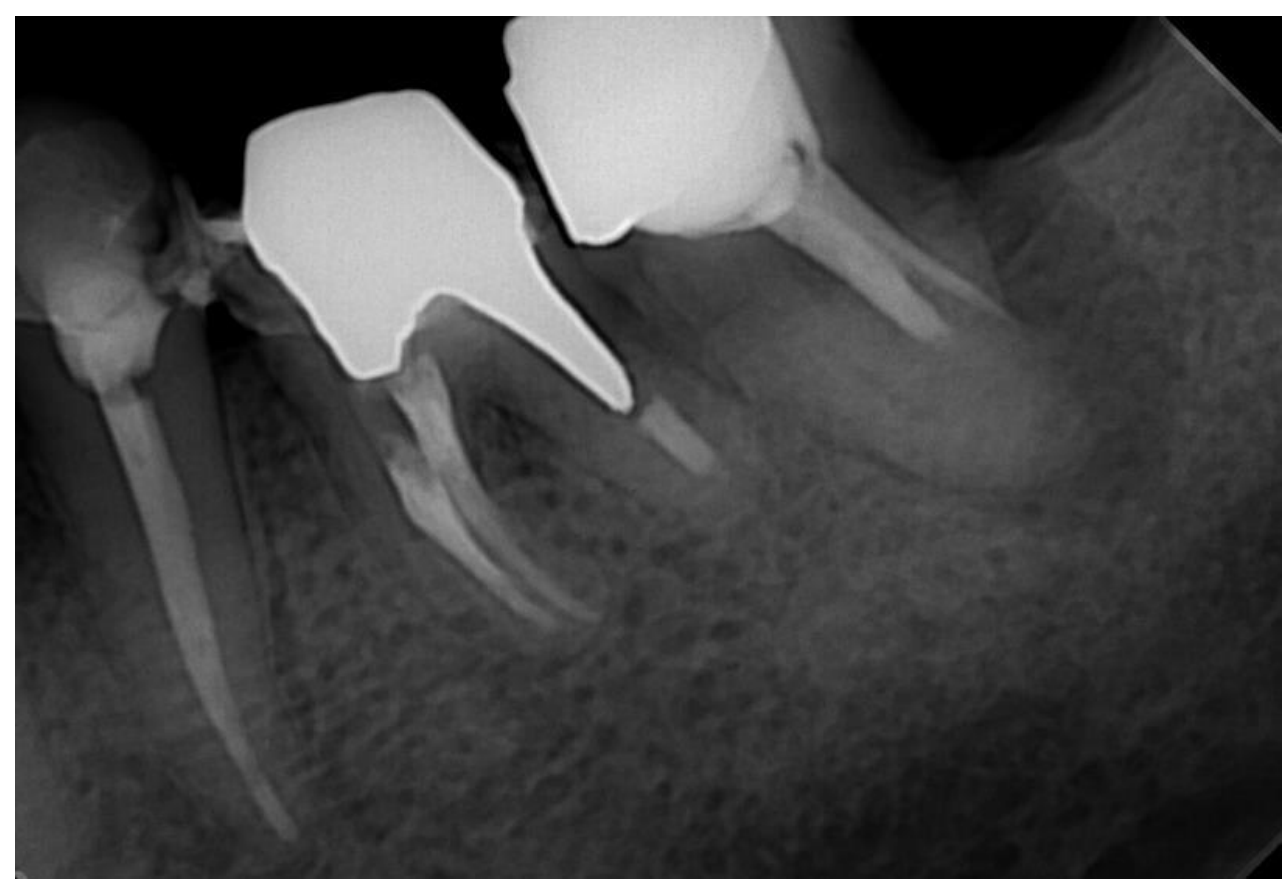

Figura 1 - Radiografia de diagnóstico do dente 37.

Confirmado o diagnóstico de Periodontite Apical Assintomática o tratamento foi iniciado sob isolamento absoluto com a remoção parcial da restauração metálica fundida e completa do cimento presente na câmara pulpar e entrada dos canais, possibilitando observar a presença de guta-percha e a entrada dos canais configurando a anatomia em C. Foram detectados um canal mésio-vestibular sem qualquer sinal de manipulação e um que iniciava na região mésio lingual estendendo-se em curva até a região disto-vestibular. Em seguida foi realizada a remoção do material obturador do canal distal e o preparo do terço cervical dos com as limas 30.10 do Prodesign $S$ (Easy Equipamentos 
Odontológicos, Belo Horizonte, Brasil) utilizando como substância química irrigadora o hipoclorito de sódio a 2,5\% e, em seguida, procedeu-se a odontometria com auxílio do aparelho Root ZX II (J. Morita, Kyoto, Japão), confirmada radiograficamente (Figura 2).

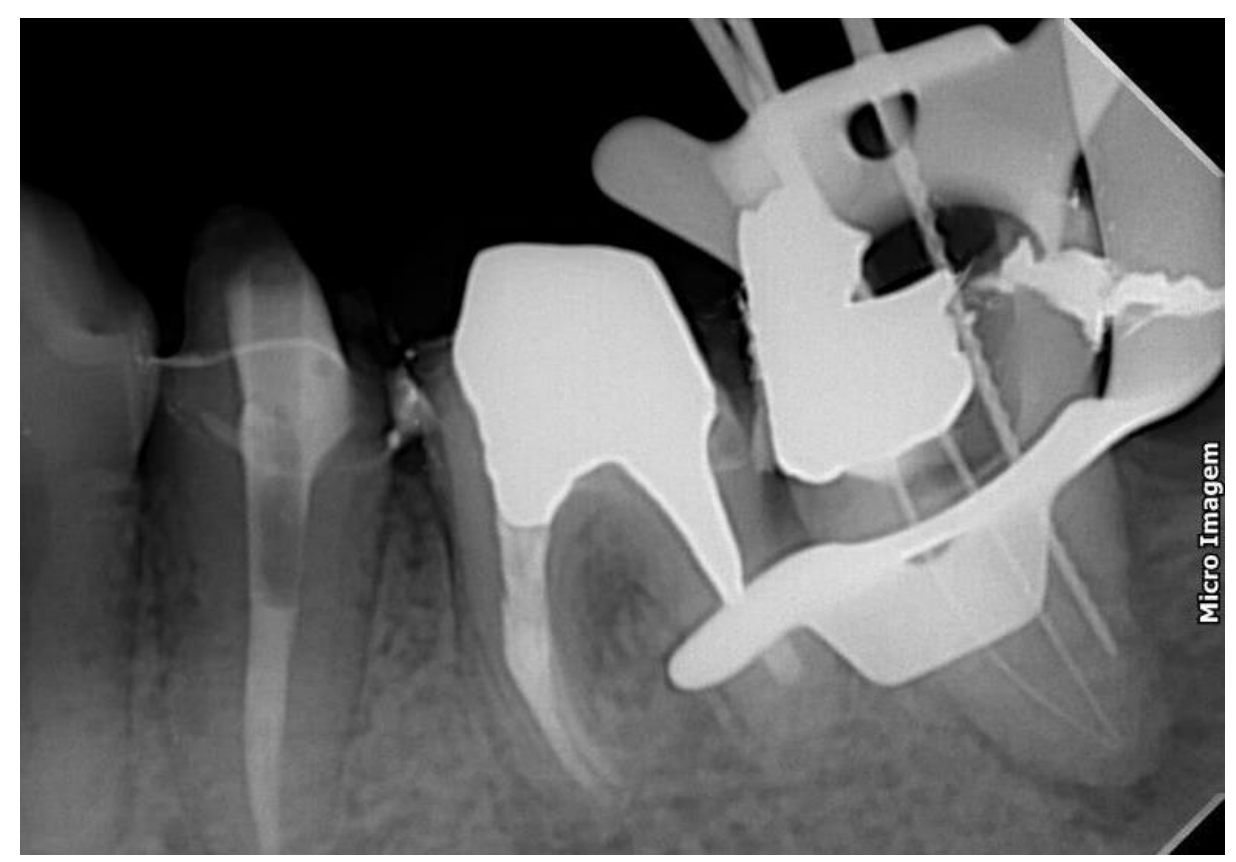

Figura 2 - Radiografia de odontometria.

Durante a odontometria foi possível confirmar a bifurcação do canal distal onde a ramificação mesial apresentava o mesmo forame do canal mésio vestibular e o distal apresentava um forame amplo, compatível com lima 70. O preparo químico cirúrgico foi desenvolvido com limas Prodesign $S^{\circledR} 25.08$ no canal mesio vestibular e, no canal em forma de $\mathrm{C}$, além desta lima, foram utilizadas limas manuais tipo $\mathrm{K}$ em sequência de 15 a 40 na ramificação mesial e 15 a 80 no canal distal, com auxílio de hipoclorito de sódio a 2,5\%. Ao final do preparo utilizou-se EDTA-T 17\% e hipoclorito de sódio a 2,5\% sob agitação com auxílio de Easy Clean (Easy Equipamentos Odontológicos, Belo Horizonte, Brasil) por 30 segundos três vezes cada,os canais foram secos e foi inserido medicação à base de hidróxido de cálcio Ultracall XS (ULTRADENT, South Jordan, EUA). Após 30 dias o paciente retornou ao consultório onde foi examinado clínica e radiograficamente (Figura 3). Através de sondagem periodontal foi observada a profundidade de sondagem máxima de $3 \mathrm{~mm}$ na distal do dente 37 . 


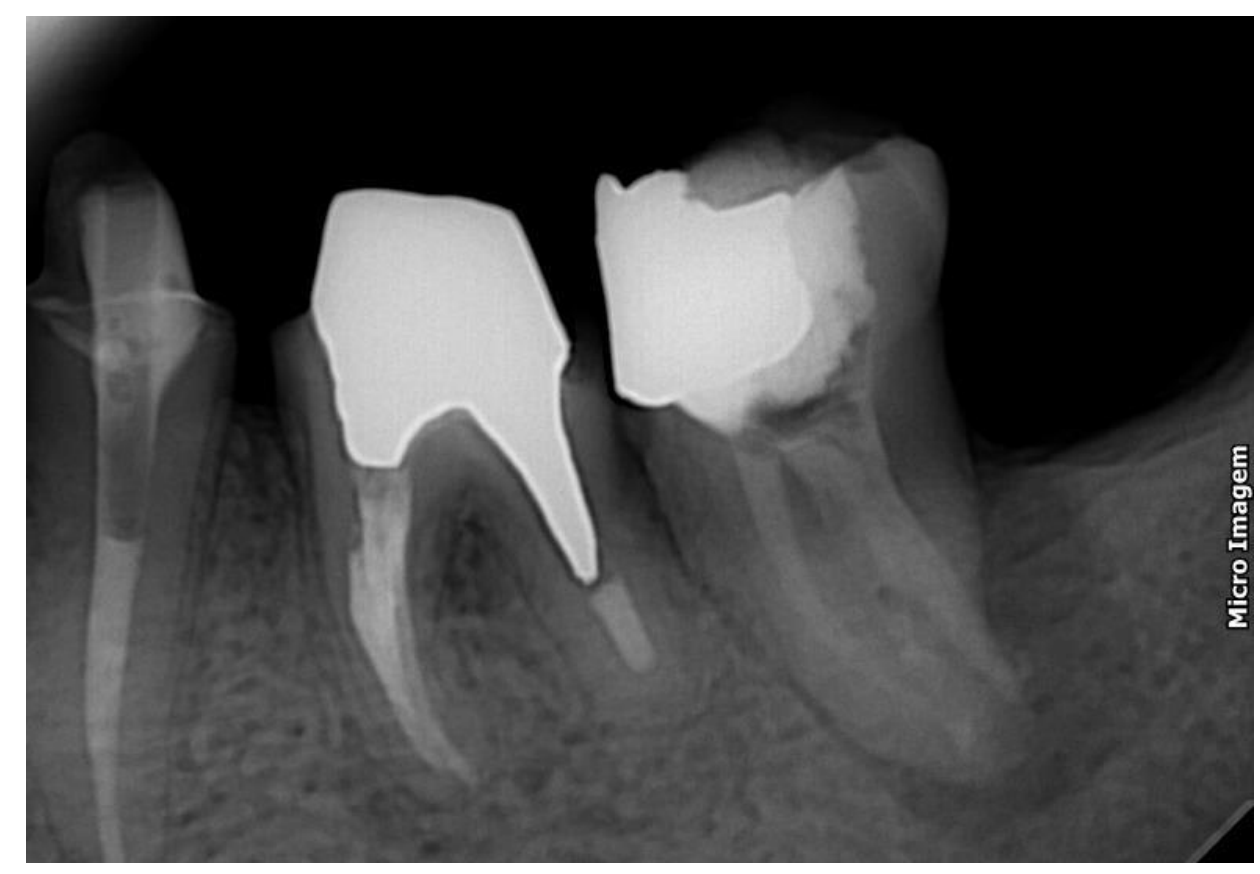

Figura 3 - Radiografia prévia a obturação

Iniciou-se então a remoção do selamento provisório e, sob isolamento absoluto, a remoção da medicação intracanal com auxílio de limas manuais e EDTA-T 17\%. O canal mesio vestibular foi obturado com guta-percha e cimento Endofill (Dentsply, Petrópolis, Brasil) e devido a presença do forame amplo no canal em forma de $\mathrm{C}$ optou-se pela obturação deste canal com MTA (Angelus, Londrina, Brasil) (Figuras 4 e 5). Foi preparado o espaço para pino no canal em forma de $\mathrm{C}$ e o dente selado provisoriamente e encaminhado ao indicador para reabilitação.

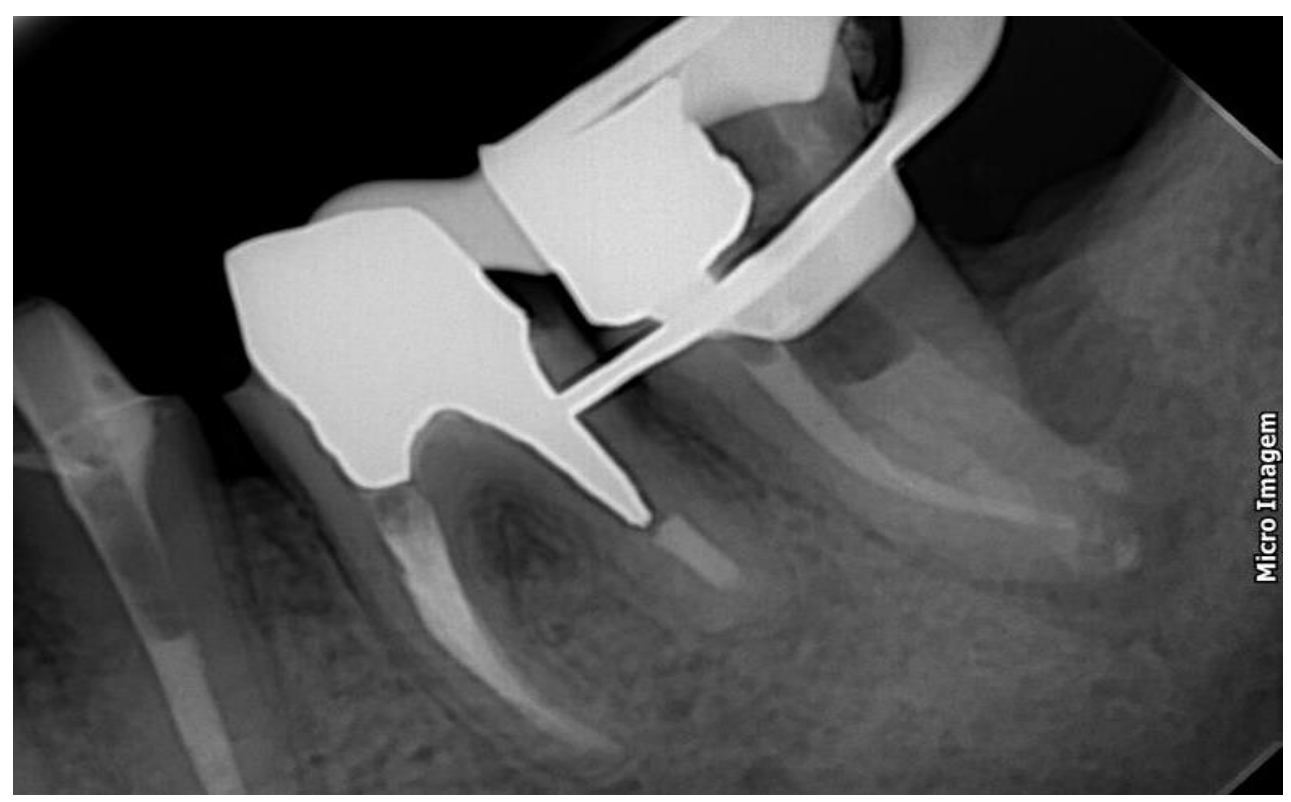

Figura 4 - Radiografia de avaliação da obturação 


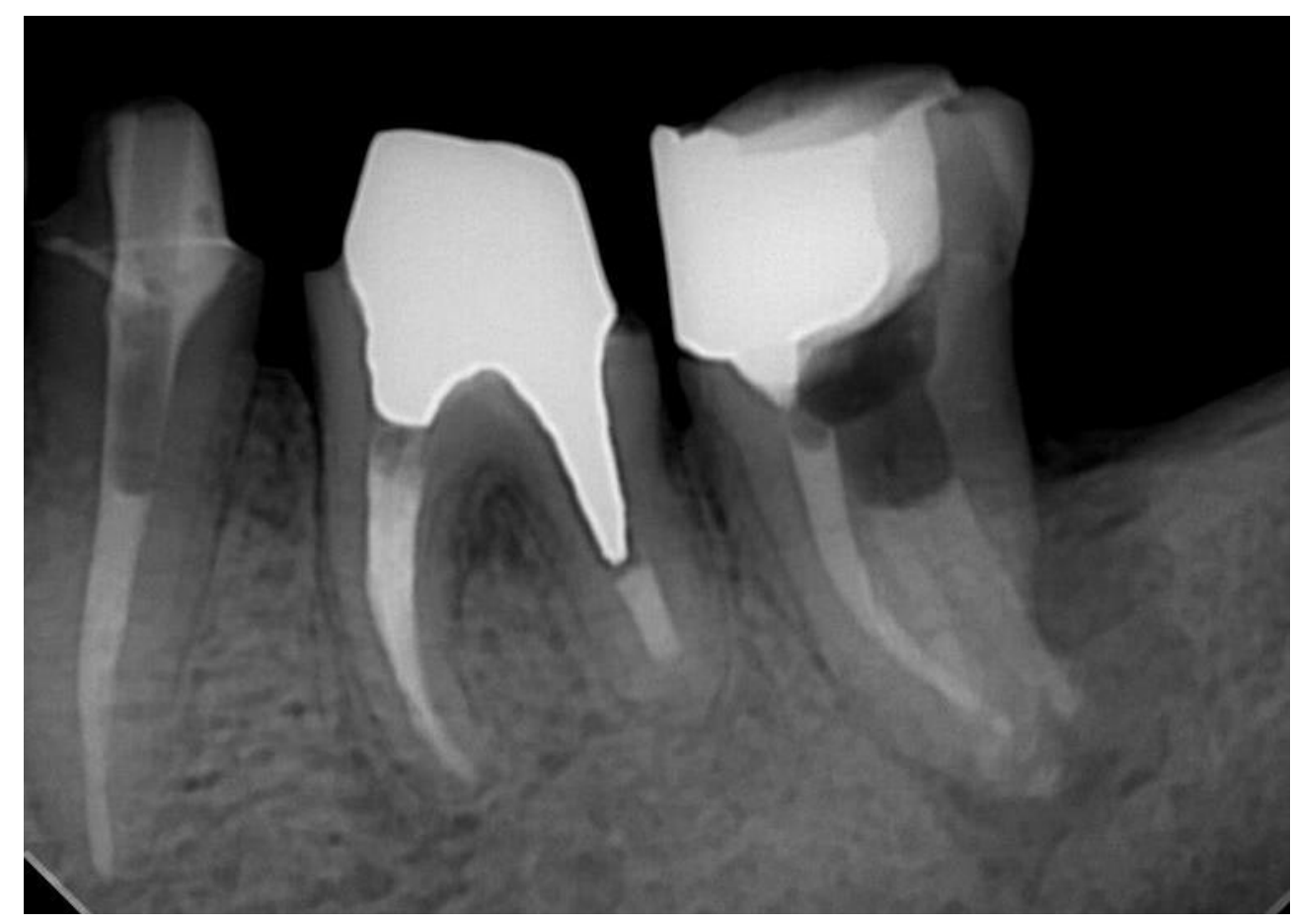

Figura 5 - Radiografia final

O paciente retornou para consultas de proservação após 45 dias (Figura 5), 75 dias (Figura 6), 120 dias (Figura 7) e 17 meses após a obturação (Figura 8). Em todas as consultas de acompanhamento o dente apresentava-se assintomático, sem a presença de alteração clinica periodontal e periapical.

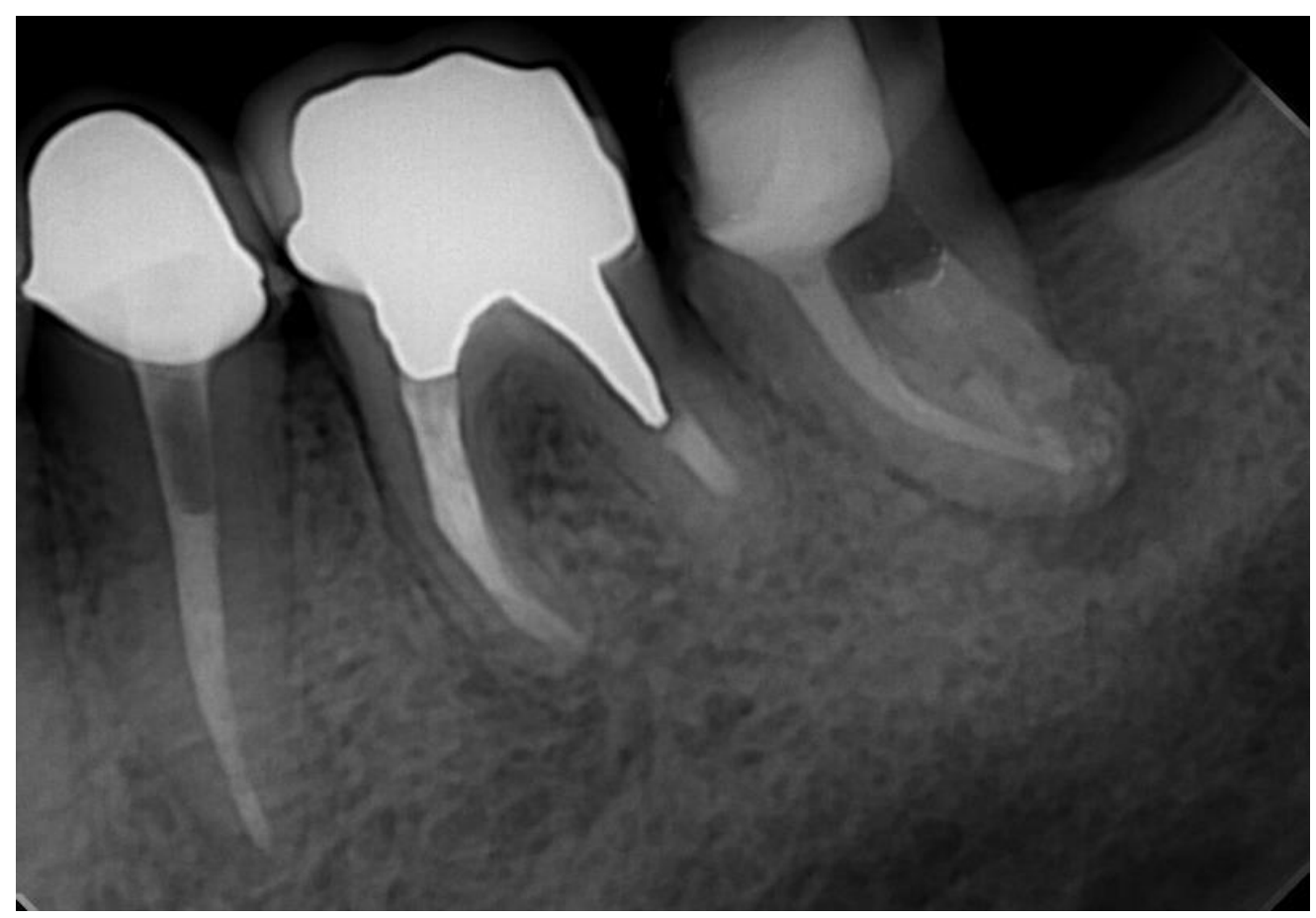

Figura 6 - Radiografia de proservação após 45 dias 


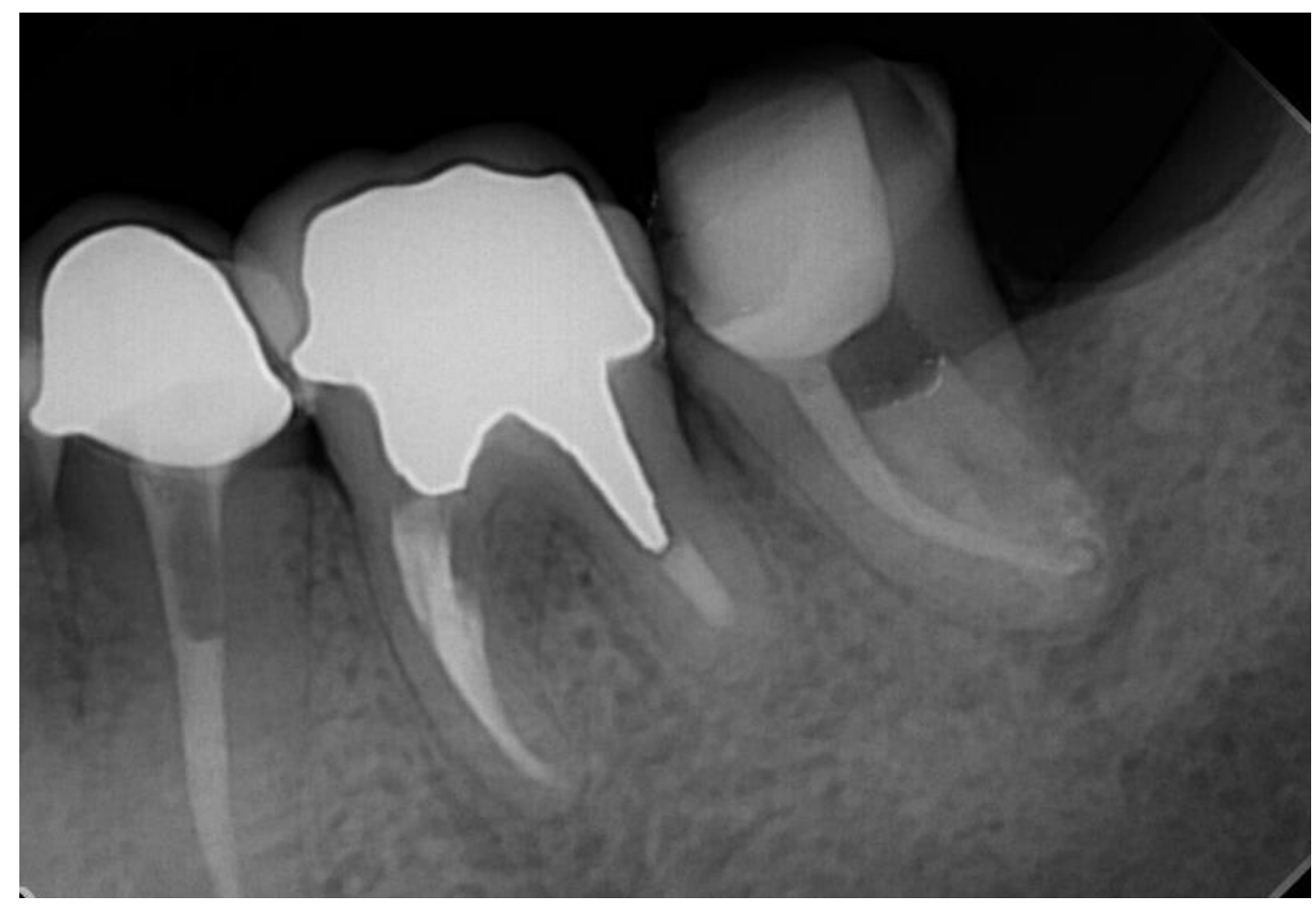

Figura 7 - Radiografia de proservação após 75 dias

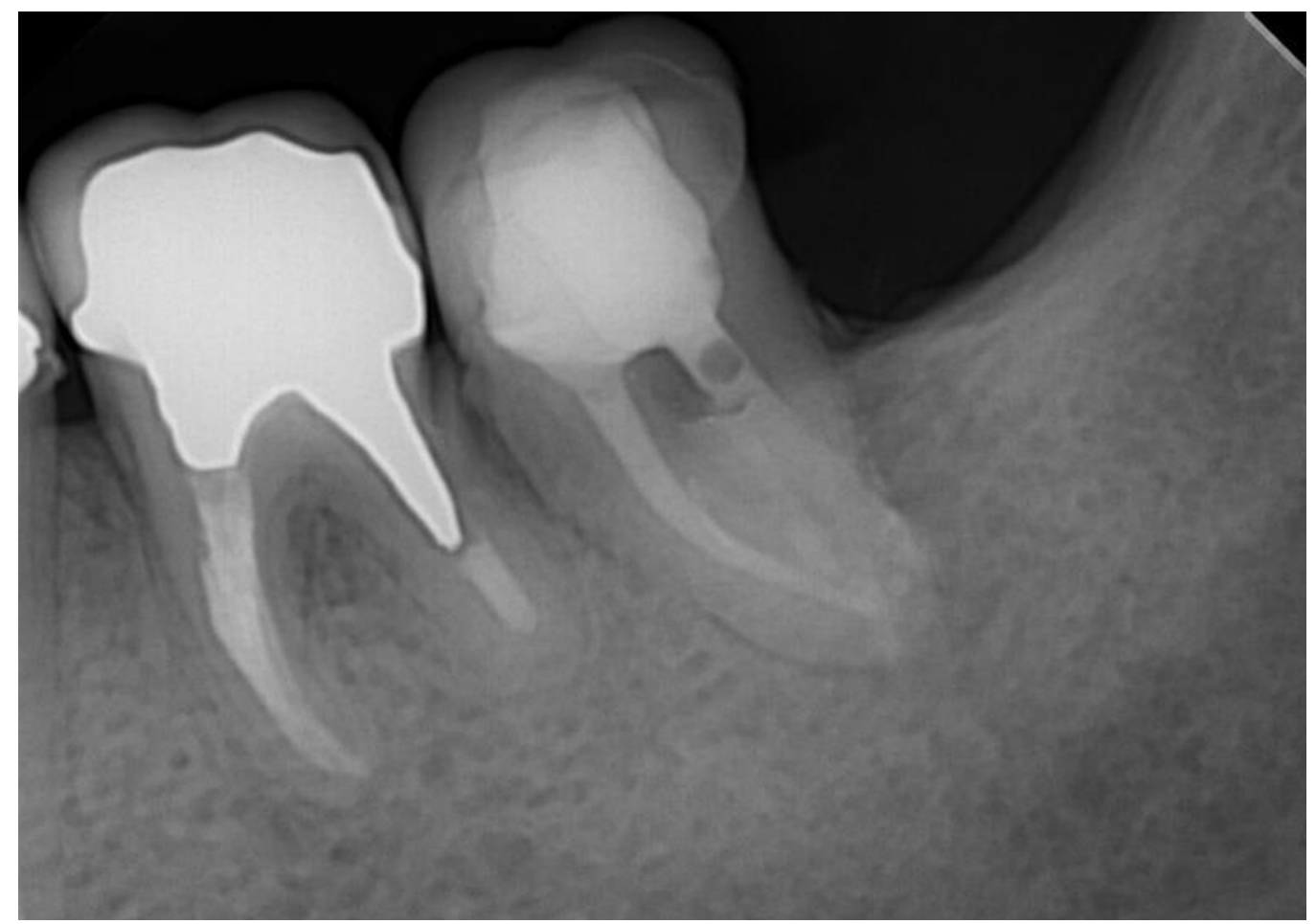

Figura 8 - Radiografia de proservação após 120 dias 


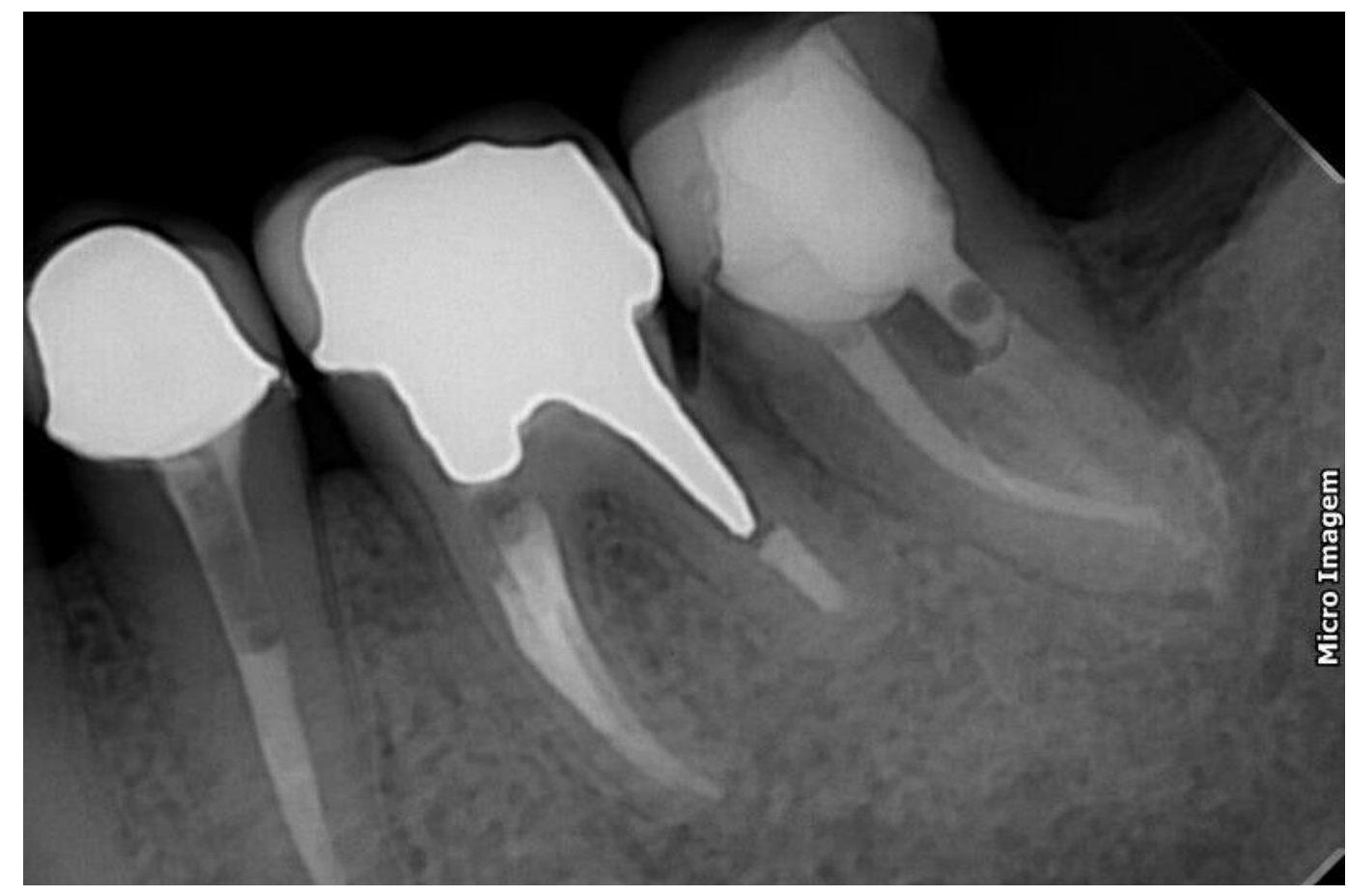

Figura 9 - Radiografia de proservação após 17 meses

\section{DISCUSSĀO}

A possibilidade da presença de canais em forma de $C$ deve ser considerada durante o exame clínico e radiográfico do paciente. O reconhecimento precoce dessas conformações facilita a limpeza, modelagem e obturação do sistema de canais radiculares 1. Embora Cook e Cox ${ }^{2}$ relatarem em seu estudo a impossibilidade de se diagnosticar canais em forma de C pela radiografia, em nosso relato foi possível observar, considerando que 0 dente apresentava tratamento endodôntico prévio, algumas características relacionadas a dentes com canal em forma de C relatadas na literatura ${ }^{5}$. Apesar de a maioria dos dentes em forma de C apresentarem características típicas, em alguns casos o diagnóstico é incerto e o exame clínico durante o procedimento endodôntico é necessário ${ }^{5}$, portanto, a confirmação dessa variação anatômica foi determinada após a remoção da restauração metálica.

Durante as tentativas de localizar todos os canais o desgaste excessivo das cavidades de acesso em largura e profundidade pode resultar em perfuração no assoalho pulpar ou no terço coronal da raiz. A tentativa de negociar os pequenos canais semelhantes ao istmo na área de união podem resultar em orifícios desnecessariamente grandes demais e/ou perfuração ao redor do canal principal adjacente à área de junção. 
Instrumentação excessiva pode ser focada em uma determinada área porque os canais em forma de C podem ser unidos e separados em toda sua extensão. Portanto, cuidadosa localização e negociação dos canais e o meticuloso desbridamento mecânico e químico do tecido pulpar devem ser realizados para o tratamento bem-sucedido de um canal em forma de $\mathrm{C}^{6}$. No entanto, diferentes tamanhos de preparo e conicidade de instrumentos também podem ser responsáveis por diferentes resultados ${ }^{10}$. Em nosso relato a reintervenção foi realizada com auxílio de microscópio facilitando a visualização da anatomia interna e evitando desgastes excessivos, a instrumentação foi adequada a anatomia individual dos canais instrumentados, considerando que o canal mésio vestibular não havia sido manipulado e a bifurcação do canal em forma de $\mathrm{C}$, com as ramificações mesial e distal apresentando diferentes diâmetros.

Os materiais obturadores podem ser compactados em irregularidades do canal radicular não preenchidas anteriormente durante o retratamento com limas rotatórias de $\mathrm{NiTi}{ }^{10}$, sendo assim, o canal onde havia material obturador teve sua remoção inicial com limas rotatórias, porém limas manuais foram utilizadas em sequência durante o preparo com o objetivo de melhorar a limpeza de regiões e irregularidades não alcançados pelas limas rotatórias.

A remoção do material obturador é um pré-requisito para o retratamento não cirúrgico, pois permite a limpeza, modelagem e a obturação subsequente do sistema de canais radiculares ${ }^{11}$. Não é comprovado que a remoção de todo o material de obturação garantirá o sucesso do retratamento endodôntico e que a guta-percha ou o cimento restante fará com que a reintervenção falhe. No entanto, remover o máximo possível de cimento e guta-percha de sistemas de canais radiculares inadequadamente preparados e obturados parece essencial para descobrir tecidos necróticos ou bactérias remanescentes que podem ser responsáveis pela inflamação e falha periapicais ${ }^{12}$.

Considerando que a anatomia nos terços médio e apical de canais em forma de $\mathrm{C}$ não pode ser previsto com base na forma da entrada ${ }^{13}$, equipados com o conhecimento da anatomia interna, meios eficazes para limpar e modelar os canais em forma de $C$ podem ser criados ${ }^{8}$. Melton et al. ${ }^{3}$ relataram em seu estudo que as características mais marcantes dos dentes examinados histologicamente foram a quantidade de detritos e espaços em canais não instrumentados nos dentes acessados e com instrumentação prévia, principalmente nos terços médio e apical da raiz. Reentrâncias e pequena área superficial desses canais impedem o completo desbridamento usando técnicas 
tradicionais de instrumentação manual, acrescentam ainda que que a maioria dos dentes examinados em seu estudo apresentou alteração anatômica do canal ao longo da raiz, tornando o desbridamento e a preparação completoa ainda mais difícil ${ }^{3}$. Com objetivo melhorar a remoção de material, limpeza e desinfecção procuramos realizar o preparo tanto mecanizado removendo a maior parte dos detritos e, considerando o formato achatado e alongado dos canais em forma de $\mathrm{C}$, manual para direcionar a instrumentação com objetivo de tocar a maior parte das paredes internas do canal possíveis incluindo istmos e reentrâncias

De acordo com Manning ${ }^{9}$ cuidados adicionais são necessárias para limpar eficazmente todo o sistema de canais, especialmente se ele contiver detritos necróticos e bactérias em suas irregularidades. A eficácia dos procedimentos de limpeza em relação aos canais radiculares em forma de $C$ ainda não foi investigada ${ }^{9}$, entretanto, a literatura mostra a importância do uso de substâncias químicas como o hipoclorito ${ }^{14,15,16}$ e o EDTA ${ }^{17,18}$ com objetivo melhorar a limpeza e a desinfeção do sistema de canais radiculares tais como o uso de mecanismos que promovem a agitação dessas substâncias ${ }^{19,20}$. Durante o tratamento optamos por utilizar como substância irrigadora o hipoclorito de sódio devido a capacidade de desinfecção e remoção de matéria orgânica além do EDTA com o objetivo de remoção de smear layer e, para potencializar a ação dessas substâncias químicas foi utilizado o Easy Clean em motor rotatório com movimento reciprocante.

A literatura específica é divergente com relação ao número de sessões necessárias para o tratamento endodôntico com dentes com necrose pulpar e lesão periapical crônica e quanto a necessidade de uso de um curativo de demora entre as sessões. Independente do número de sessões é fato que o controle bacteriológico específico do sistema de canais é crítico para o sucesso da terapia endodôntica ${ }^{21}$. Estudos mostram as boas propriedades do hidróxido de cálcio como medicação intracanal tais como o efeito antimicrobiano e a biocompatibilidade ${ }^{22,23,24}$. Procurando aumentar desinfecção intracanal possibilitando assim reparo periapical optamos por utilizar o hidróxido de cálcio como medicação intracanal por 30 dias. Foi possível observar ao exame radiográfico a extrusão não proposital do material na primeira sessão visto que o forame do canal distal estava amplo.

Durante a obturação devido a condição anatômica do forame do canal distal optamos por utilizar MTA em metade do comprimento radicular do canal em forma de C visto que o material apresenta excelentes resultados quando utilizado em dentes com 
ápice aberto ${ }^{25,26}$. O canal mésio vestibular foi obturado com cimento e guta-percha através da técnica da condensação vertical por ondas contínuas.

A associação dos fatores essenciais ao sucesso endodôntico permite resumi-los em: silêncio clínico (ausência de dor, edema, fístula), estrutura óssea periapical normal (uniformidade da lâmina dura, espaço periodontal normal, ausência ou redução de rarefação óssea, ausência ou interrupção de reabsorção radicular), dente em função e presença de selamento coronário perfeito ${ }^{27}$. O otimismo cauteloso parece mais apropriado ao prognosticar o sucesso de tratamento do canal radicular de um segundo molar inferior com um canal em forma de $\mathrm{C}^{3}$. As dificuldades encontradas frente a uma reintervenção tendem a serem maiores que quando na intervenção, talvez seria sensato o otimismo ser ainda mais cauteloso. A proservação a médio e longo prazo trará ao profissional a verdadeira resposta sobre o sucesso ou insucesso da reintervenção.

\section{CONCLUSAOO}

A complexidade anatômica interna em dentes com canais em forma de $C$ exige do profissional, além do planejamento adequado, conhecimento, destreza e um maior cuidado na condução do tratamento. A proservação dos tratamentos realizados é de fundamental importância para determinar o sucesso ou insucesso. Frente a isso, com a proservação obtida até o momento, foi possível observar o reparo ósseo associado a ausência de sinais e sintomas, e assim podemos considerar que o sucesso do tratamento foi atingido.

\section{REFERÉNCIAS}

1 Al-Fouzan KS. C-shaped root canals in mandibular second molars in a Saudi Arabian population. Int Endod J.2002;35:499-504.

2 Cooke HG, Cox FL. C-shaped canal configurations in mandibular molars. The Journal of the American Dental Association.1979;99(5):836-839.

3 Melton DC. Krell KV, Fuller MW. Anatomical and histological features of C-shaped canals in mandibular second molars. J Endod.1991;17:384-8

4 Woelber JP, Bruder M, Tennert C, Wrbas KT. Assessment of endodontic treatment of Cshaped root canals. Swiss dental journal. 2014;124:11-5. 
5 Haddad GY, Nehme WB, Ounsi HF. Diagnosis, classification, and frequency of Cshaped canals in mandibular second molars in the Lebanese population. Journal of Endodontics. 1999;25(4), 268-271:

6 Jin, GC, Lee SJ, Rob BD. Anatomical study of C-Shaped canals in mandibular second molars by analysis of computed tomography. J Endod. 2006;32(1):10-13.

7 Mashyakhy MH, Chourasia HR, Jabali AH, Bajawi HA, Jamal H, Testarelli L et al. Cshaped canal configuration in mandibular premolars and molars: Prevalence, correlation, and differences: An In Vivo study using cone-beam computed tomography. Nigerian journal of clinical practice. 2020;23(2): 232-9.

8 Fan B, Cheung GSP, Fan M, Gutmann JL, Fam W. C-Shaped canal system in mandibular second molars: part II-radiographic features. J Endod. 2004;30(12):904-8.

9 Manning SA. Root canal anatomy of mandibular second molars. Part II C-shaped canals Int Endod J. 1990;23(1):40-45.

10 Rechenberg DK, Paqué F. Impact of cross-sectional root canal shape on filled canal volume and remaining root filling material after retreatment. Int Endod J. 2012;46(6):547555 .

11 Mollo A, Botti G, Prinicipi Goldoni N, Randellini E, Paragliola R, Chazine M et al. Efficacy of two $\mathrm{Ni}$-Ti systems and hand files for removing gutta-percha from root canals. Int Endod J. 2011;45(1):1-6.

12 Schirrmeister JF, Wrbas KT, Schneider FH, Altenburger MJ, Hellwig E. Effectiveness of a hand file and three nickel-titanium rotary instruments for removing gutta-percha in curved root canals during retreatment. Oral Surgery, Oral Medicine, Oral Pathology, Oral Radiology, and Endodontology. 2006;101(4):542-547.

13 Fan B, Cheung GSP, Fan M, Gutmann JL, Bian Z. C-shaped canal system in mandibular second molars: parte I - Anatomical features. J Endod. 2004;30(12):889-903.

14 Okino LA, Siqueira EL, Santos M, Bombana AC, Figueiredo JAP. Dissolution of pulp tissue by aqueous solution of chlorhexidine digluconate and chlorhexidine digluconate gel. Int Endod J. 2004;37(1):38-41.

15 Estrela C, Silva JA, Alencar AHG, Leles CR, Decurcio DA. Efficacy of sodium hypochlorite and chlorhexidine against Enterococcus faecalis: a systematic review. Journal of Applied Oral Science. 2008;16(6):364-368

16 Haapasalo M, Shen Y, Qian W, Gao Y. Irrigation in Endodontics. Dental Clinics of North America. 2010;54(2),291-312.

17 Mello I, Robazza CRC, Antoniazzi JH, Coil J. Influence of different volumes of EDTA for final rinse on smear layer removal. Oral Surgery, Oral Medicine, Oral Pathology, Oral Radiology, and Endodontology. 2008;106(5): e40-e43. 
18 Hameed S, Ibrahim A, Al-Nasrawi S. Comparative study to evaluate the antimicrobial effect of MTAD, 17\% EDTA, and $3 \% \mathrm{NaOCl}$ against Enterococcus faecalis in primary teeth for root canals therapy (in vitro study). Medico-Legal Update. 2020; 1(20):340-5.

19 Kato AS, Cunha RS, da Silveira Bueno CE, Pelegrine RA, Fontana CE, de Martin AS. Investigation of the efficacy of Passive Ultrasonic Irrigation versus irrigation with Reciprocating Activation: An environmental Scanning Electron Microscopic Study. J Endod. 2016;42(4):659-663.

20 Singh R, Nikhil V, Jaiswal S, Gupta S, Raj S, Arora R. A comparative evaluation of the efficacy of activated irrigation protocol on root canal debridement - A scanning electron microscopic study. Endodontology. 2020;32(1):26-23.

21 Silva RAB, Silva LAB, Nelson-Filho P. O emprego do hidróxido de cálcio (pasta Calen) como medicação tópica entre sessões (curativo de demora). In Leonardo, M.R.; Leonardo, R.T. Endodontia: Conceitos Biológicos e Recursos Tecnológicos. São Paulo: Artes Médicas; 2009.p225-254.

22 Zmener O, Pameijer $\mathrm{CH}$, Banegas $\mathrm{G}$. An in vitro study of the $\mathrm{pH}$ of three calcium hydroxide dressing materials. Dental Traumatology. 2007;23(1):21-25.

23 Blanscet ML, Tordik PA, Goodell GG. An Agar diffusion comparison of the antimicrobial effect of calcium hydroxide at five different concentrations with three different vehicles. $J$ Endod. 2008;34(10):1246-1248.

24 Andolfatto C, da Silva GF, Cornélio ALG, Guerreiro-Tanomaru JM, Tanomaru-Filho M, Faria $\mathrm{G}$ et al. Biocompatibility of intracanal medications based on Calcium Hydroxide. ISRN Dentistry, 2012;1-6.

25 Alkahtani A, Shostad S, Schifferle R, Bhambhani S. In-vitro evaluation of microleakage of an orthograde apical plug of Mineral Trioxide Aggregate in permanent teeth with simulated immature apices. J Endod. 2005;31(2):117-119.

26 Martin R, Monticelli F, Brackett W, Loushine R, Rockman R, Ferrari M et al.Sealing properties of Mineral Trioxide Aggregate orthograde apical plugs and root fillings in an In vitro apexification model. J Endod. 2007;33(3):272-275.

27 Pereira Junior W, Moura MS, Guedes AO, Decurcio RA, Estrela C. Análise de critérios de sucesso em Endodontia e Implantodontia. Rev Odontol Bras Central. 2010;19(49):10818. 